\title{
Comportamiento poscosecha de frutos de mandarina (Citrus reticulata Blanco) var. Arrayana: efecto de diferentes tratamientos térmicos
}

\section{Postharvest behavior of mandarin fruits (Citrus reticulata Blanco) var. Arrayana: effect of different thermal treatments}
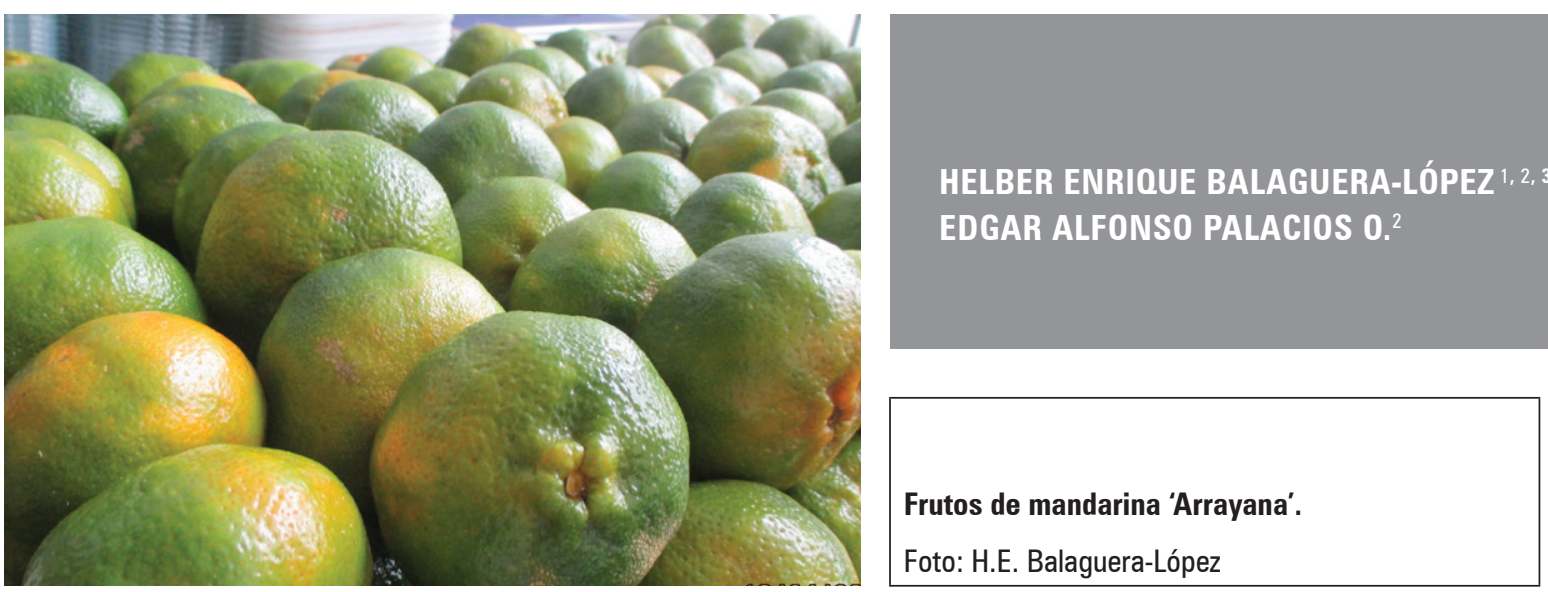

\section{RESUMEN}

La mandarina (Citrus reticulata Blanco) 'Arrayana' es una de las variedades más importantes en el país, sin embargo, las pérdidas en poscosecha son altas por la alta perecibilidad del fruto. Por tanto, el objetivo de este estudio fue evaluar el efecto de diferentes tratamientos térmicos sobre el comportamiento poscosecha de frutos de mandarina var. Arrayana almacenados a $2^{\circ} \mathrm{C}$ durante 40 días. Se utilizó un diseño completamente al azar, donde se evaluaron los siguientes tratamientos térmicos: (T1) frutos testigo, (T2) frutos tratados con agua caliente a $50^{\circ} \mathrm{C}$ durante 5 minutos, (T3) frutos tratados con agua caliente a $53^{\circ} \mathrm{C}$ durante 3 minutos, (T4) frutos sometidos a calentamiento intermitente con ciclos de 8 días a $2^{\circ} \mathrm{C}+1$ día a $18^{\circ} \mathrm{C}$ y (T5) frutos sometidos a calentamiento intermitente con ciclos de 12 días a $2^{\circ} \mathrm{C}+1$ día a $18^{\circ} \mathrm{C}$. Durante el almacenamiento no se presentaron síntomas de daños por frío visibles. Al final de almacenamiento, no hubo diferencias estadísticas en los azúcares sacarosa, fructosa y glucosa, la acidez total titulable, los ácidos cítrico, málico y ascórbico ni los sólidos solubles totales. El calentamiento intermitente se caracterizó por aumentar las pérdidas de peso de los frutos y la tasa respiratoria, pero generó mejor coloración de los frutos porque indujo mayor cambio de color de la epidermis al presentar mayor acumulación de carotenoides totales y una baja concentración de clorofilas.

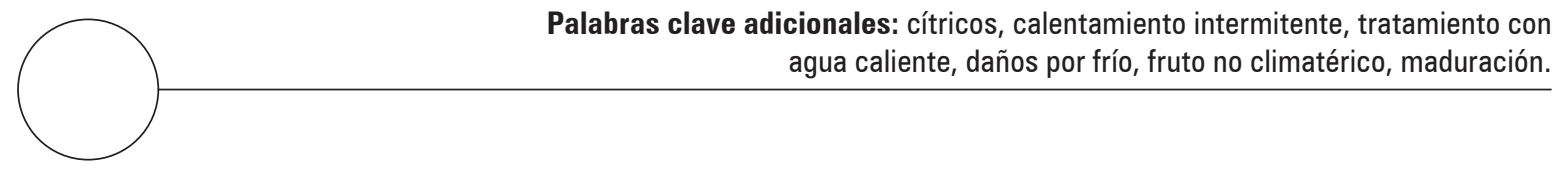

Facultad de Ciencias Agropecuarias, Grupo de Investigaciones Agrícolas, Universidad Pedagógica y Tecnológica de Colombia (UPTC), Tunja (Colombia). ORCID Balaguera-López, H.E.: 0000-0003-3133-0355

2 Facultad de Ciencias, Programa de Biología, Grupo de Investigación de Biología de la Universidad El Bosque (GRIB), Universidad El Bosque, Bogotá (Colombia). ORCID Palacios O., E.A.: 0000-0003-3349-824X

3 Autor para correspondencia.hbalaguera@unbosque.edu.co 


\section{ABSTRACT}

Mandarin (Citrus reticulata Blanco) 'Arrayana' is one of the most important varieties in the country; however, postharvest losses are high as a result of the high perishability of the fruit. Therefore, the objective of this study was to evaluate the effect of different thermal treatments on the postharvest behavior of mandarin fruits var. Arrayana stored at $2^{\circ} \mathrm{C}$ for 40 days. A completely randomized design was used, where the following thermal treatments were evaluated: (T1) control fruits, (T2) fruits treated with hot water at $50^{\circ} \mathrm{C}$ for 5 minutes, (T3) fruits treated with hot water at $53^{\circ} \mathrm{C}$ for 3 minutes, (T4) fruits subjected to intermittent warming with cycles of 8 days at $2^{\circ} \mathrm{C}+1$ day at $18^{\circ} \mathrm{C}$ and (T5) fruits subjected to intermittent warming with cycles of 12 days at $2^{\circ} \mathrm{C}+1$ day at $18^{\circ} \mathrm{C}$. During storage there were no visible symptoms of chilling injury. At the end of storage, there were no statistical differences in sucrose, fructose and glucose, titratable acidity, citric, malic and ascorbic acids or total soluble solids. The intermittent warming was characterized by increasing fruit weight loss and respiratory rate, but it generated better fruit coloration because it induced a greater color change of the epidermis when there was a greater accumulation of total carotenoids and a low concentration of chlorophylls.

Additional key words: citrics, intermittent warming, hot water treatment, chilling injury, non-climacteric fruit, ripening.

Fecha de recepción: 03-02-2018 Aprobado para publicación: 30-05-2018
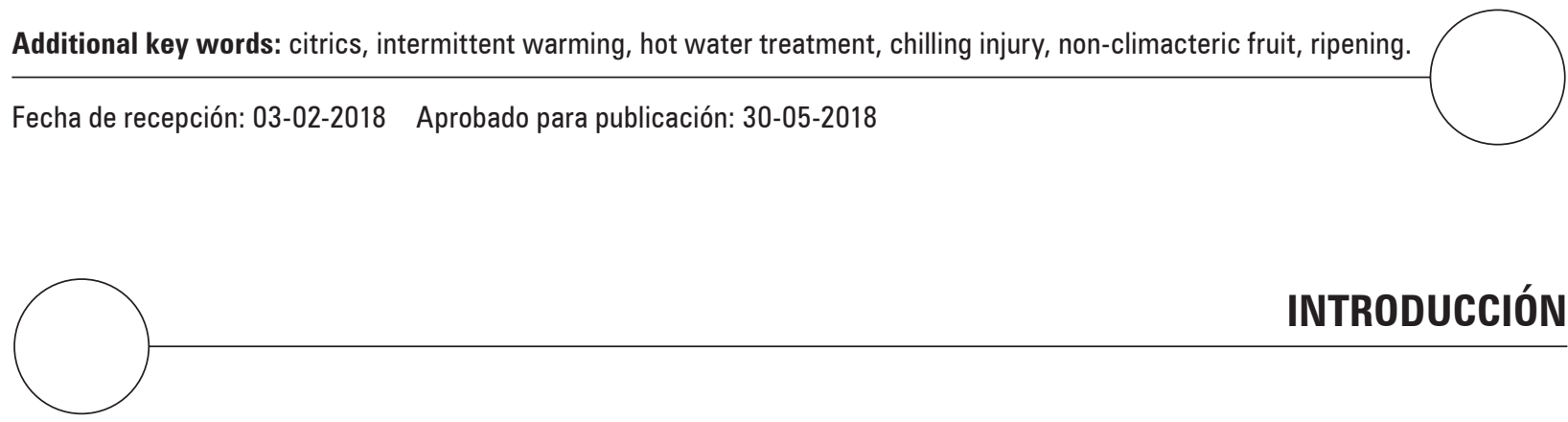

INTRODUCCIÓN

La mandarina 'Arrayana' es la variedad que más se cultiva en Colombia (Ordúz-Rodríguez et al., 2012; Chaparro-Zambrano et al., 2017). Es una variedad adaptada a trópico bajo, su fruto es reconocido por sus atributos gustativos, su apariencia externa y comercial (Ordúz-Rodríguez et al., 2012). En Colombia, los cítricos, incluyendo la mandarina presentan altas pérdidas en poscosecha que están entre un $12 \%$ a $25 \%$, lo cual se debe, entre otros factores, al deficiente manejo poscosecha que existe en el país, por tanto, para reducir las pérdidas de frutos es importante comprender los factores de tipo biológico y ambiental implicados en la degradación del fruto, así como conocer y utilizar la tecnología poscosecha más adecuada para retrasar la senescencia del producto y mantener la calidad por más tiempo (Carvalho et al., 2012).

La conservación en la que se emplea baja temperatura es la tecnología más utilizada y eficaz para disminuir el deterioro poscosecha de productos hortofrutícolas, ya que reduce las pérdidas comerciales durante el almacenamiento (Lado et al., 2015). Sin embargo, los cítricos presentan susceptibilidad a las bajas temperaturas causando alteraciones o manchas en la epidermis, estos cambios, se les conocen como daños por frío y pueden generar altas pérdidas de calidad comercial (Carvalho et al., 2012). En general los frutos de las diferentes especies de cítricos y cultivares son sensibles a daños por frío cuando se almacenan por debajo de $10^{\circ} \mathrm{C}$ (Lafuente y Zacarías, 2006). Al respecto, Ladaniya (2008) recomienda temperaturas de almacenamiento para mandarinas 'Ellendale', 'Murcott' y 'Nagpur' entre 6 y $7^{\circ} \mathrm{C}$.

Para evitar los daños por frío es recomendable el almacenamiento a temperaturas por encima del umbral para evitar el desarrollo de esta fisiopatía. El problema, es que esto limita la supervivencia comercial del producto, al estar almacenado a una temperatura relativamente elevada para ralentizar los procesos fisiológicos, por tal razón, una solución viable es la aplicación de tratamientos térmicos que disminuyan la sensibilidad de los frutos de mandarina a los daños por frío durante o después del almacenamiento a bajas temperaturas. Se han reportado varios tratamientos poscosecha para reducir los síntomas de daños por frío, estos incluyen el calentamiento intermitente, el vapor y el agua caliente (Sala y Lafuente, 2000; Fallik, 2004; Liu et al., 2015).

El calentamiento intermitente es un tratamiento que consiste en refrigerar los frutos y periódicamente llevarlos a otro ambiente de mayor temperatura durante unas horas o días, con el fin de hacer reversible los 
procesos iniciales de daños por frío a nivel fisiológico que ya han ocurrido, pero que no se han manifestado en forma de síntomas. Posteriormente, se vuelve a bajar la temperatura para beneficiarse de la refrigeración en la supervivencia del producto (Artés y Artés-Hernández, 2002). Este tratamiento ha sido utilizado con resultados favorables en naranja 'Navelate' (Artés et al., 1998). No obstante, Crisosto et al. (2008) mencionan que un calentamiento intermitente incorrecto evita que el fruto recupere sus funciones metabólicas, generando una senescencia acelerada. Esto indica la importancia de encontrar una recomendación precisa a la hora de llevar a cabo el calentamiento intermitente.

Por su parte, los tratamientos térmicos mediante inmersión en agua caliente también son eficaces para inducir tolerancia al frío en frutos, mediante la modulación de los sistemas antioxidantes, encargados de prevenir la acumulación de especies reactivas de oxígeno (Sala y Lafuente, 2000; Ma et al., 2014). Este tipo de tratamiento puede aumentar la producción y activación de proteínas de choque térmico tal como lo indican Rozenzvieg et al. (2004), y algunas de estas proteínas pueden actuar como chaperonas, involucradas en el plegamiento, ensamblaje y transporte de proteínas (Rozenzvieg et al., 2004), muchas de las cuales están presentes y tienen funciones biológicas en la membrana celular, evitando de esta manera un mayor daño aún en condiciones de bajas temperaturas en este importante organelo celular. Investigadores como Ghasemnezhad et al. (2008) en mandarina 'Satsuma', encontraron resultados favorables con agua a $47,5^{\circ} \mathrm{C}$ por 2 o $5 \mathrm{~min}$ y con agua a $50^{\circ} \mathrm{C}$ por 2 min. De acuerdo con Holland et al. (2012) tiempos prolongados y altas temperaturas del tratamiento térmico con agua pueden generar efectos negativos en la calidad de los frutos. No obstante, tanto el tiempo como la temperatura del tratamiento dependerá de la especie, el tamaño, estado de madurez, entre otros (Ma et al., 2014).

En este estudio se quiere hacer énfasis en el efecto que puedan tener los tratamientos térmicos sobre el comportamiento poscosecha de los frutos, es así como Shadmani et al. (2015) mencionan el carácter de los tratamientos térmicos en el retraso del proceso de maduración de los frutos. Al respecto, Ghasemnezhad et al. (2008) reportan que en mandarina 'Satsuma', el tratamiento con agua caliente a 50 o $55^{\circ} \mathrm{C}$ por 2 min disminuyó la tasa respiratoria y también la producción de etileno, pero no afectó la acidez. Mientras que Schirra et al. (2004) observaron que el tratamiento térmico con agua caliente no afectó los sólidos solubles totales, la acidez total titulable y el ácido ascórbico en naranja 'Valencia'. Por lo anterior, el objetivo de este estudio fue evaluar el efecto de diferentes tratamientos térmicos sobre el comportamiento poscosecha de frutos de mandarina (Citrus reticulata Blanco) var. Arrayana almacenados a $2^{\circ} \mathrm{C}$.

\section{MATERIALES Y MÉTODOS}

Los frutos de mandarina (Citrus reticulata Blanco) 'Arrayana' fueron obtenidos de cultivos comerciales del municipio de Apulo, Cundinamarca (Colombia). Los frutos fueron recolectados en madurez de cosecha, con los parámetros que se muestran en la tabla 1. El experimento se realizó en el Laboratorio de Investigación en Biología de la Universidad El Bosque y en el Laboratorio de Fisiología Vegetal de la Universidad Pedagógica y Tecnológica de Colombia.

Tabla 1. Parámetros fisicoquímicos de los frutos de mandarina 'Arrayana' al inicio del almacenamiento.

\begin{tabular}{|l|c|}
\hline \multicolumn{1}{|c|}{ Parámetro } & Valor \\
\hline Peso $(\mathrm{g})$ & $112,77 \pm 12,76$ \\
\hline $\begin{array}{l}\text { Índice de color de la epidermis } \\
\left(\mathrm{IC}=1.000 \times \mathrm{a}^{*} / \mathrm{L}^{*} \times \mathrm{b}^{*}\right)\end{array}$ & $3,34 \pm 1,6$ \\
\hline Acidez total titulable $(\%)$ & $0,83 \pm 0,3$ \\
\hline Sólidos solubles totales $\left({ }^{\circ} \mathrm{Brix}\right)$ & $11,82 \pm 1,5$ \\
\hline Tasa respiratoria $\left(\mathrm{mg} \mathrm{CO}_{2} \mathrm{~kg}^{-1} \mathrm{~h}^{-1}\right)$ & $1,94 \pm 0,22$ \\
\hline
\end{tabular}

Promedio de 10 frutos \pm desviación estándar.

Se utilizó un diseño completamente al azar y se evaluaron los siguientes tratamientos térmicos: (T1) frutos sin tratamiento térmico, (T2) frutos tratados con agua caliente a $50^{\circ} \mathrm{C}$ durante $5 \mathrm{~min}$, (T3) frutos tratados con agua caliente a $53^{\circ} \mathrm{C}$ durante 3 min, (T4) frutos sometidos a calentamiento intermitente con ciclos de $8 \mathrm{~d}$ a $2^{\circ} \mathrm{C}+1 \mathrm{~d}$ a $18^{\circ} \mathrm{C}$ y (T5) frutos sometidos a calentamiento intermitente con ciclos de $12 \mathrm{~d}$ a $2^{\circ} \mathrm{C}+1 \mathrm{~d}$ a $18^{\circ} \mathrm{C}$. Los tratamientos con agua caliente se realizaron previo al almacenamiento refrigerado. Cada uno de los tratamientos se repitió cuatro veces, para un total de 20 unidades experimentales, y cada unidad experimental estuvo compuesta por aproximadamente $2 \mathrm{~kg}$ de fruta.

Los frutos se almacenaron a $2 \pm 0,5^{\circ} \mathrm{C}$ durante $40 \mathrm{~d}$. Periódicamente se determinaron las siguientes variables: tasa de respiración, los frutos fueron puestos en una cámara hermética de $2 \mathrm{~L}$, donde la emisión de 
$\mathrm{CO}_{2}$ producto de la respiración se determinó mediante sensores infrarrojo de $\mathrm{CO}_{2}$ acoplados a un sistema de captura de datos denominado Labquest (Vernier Software \& Technology, Beaverton, OR, USA), fue necesario registrar el peso de la muestra para finalmente expresar la respiración en mg CO $\mathrm{kg}^{-1} \mathrm{~h}^{-1}(\mathrm{Ba}-$ laguera-López et al., 2016). La pérdida de peso (\%) se realizó a través de medición de la masa fresca a los frutos en balanza de precisión con aproximación de $0,001 \mathrm{~g}$.

$\mathrm{Al}$ cabo de los $40 \mathrm{~d}$ de almacenamiento se determinó lo siguiente: índice de daños por frío, siguiendo la metodología reportada por Balaguera (2015). Para determinar carotenoides y clorofilas totales, se pesó alrededor de $1 \mathrm{~g}$ de epidermis, se agregaron $5 \mathrm{~mL}$ de acetona, se agitó en vórtex durante $1 \mathrm{~min}$ y luego se centrifugó durante $10 \mathrm{~min}$ a $4.000 \mathrm{rpm}$. Luego se ubicó el sobrenadante en un balón de $20 \mathrm{~mL}$, al pellet nuevamente se le adicionó acetona, repitiendo el anterior paso hasta que el pellet quedó incoloro. El sobrenadante se llevó a volumen de $20 \mathrm{~mL}$ con acetona, posteriormente se midió la absorbancia en espectrofotómetro (UV-ViS Genesys 10; Thermo Fisher Scientific, Waltham, MA, USA) a $450 \mathrm{~nm}$ para carotenoides y a 663 y $645 \mathrm{~nm}$ para clorofilas.

Para la firmeza $(N)$, se hicieron dos mediciones en la zona ecuatorial del fruto mediante el uso de un penetrómetro digital (PCE-PTR200; PCE Instruments, Alicante, España), con puntal de $6 \mathrm{~mm}$ y precisión de $\pm 0,5 \%$. Para el color de la epidermis, se calculó la diferencia o delta de color de acuerdo con la metodología utilizada por Mendoza et al. (2006), para lo cual, se determinaron los parámetros del sistema CIELab " $\mathrm{L}^{*}$ ", "a*" $\mathrm{y}$ " $\mathrm{b}$ " en tres puntos equidistantes del fruto mediante colorímetro digital CR-20 (Konica Minolta, Tokyo, Japón). Mediante cromatografía líquida de alta eficiencia (HPLC) se cuantificó el contenido de sacarosa, fructosa y glucosa y ácidos orgánicos (cítrico, málico, oxálico y ascórbico), se utilizó cromatógrafo HPLC Ultimate 3000 Dionex (Thermo Fisher Scientific, Waltham, MA, USA), equipado con columna Hypersil Gold Amino 250 x 4,6 $55 \mu \mathrm{m}$, detector DAD en 4 canales $(210,240,272$ y $280 \mathrm{~nm}$ ); índice de refracción $\left(35^{\circ} \mathrm{C}\right)$ y como fase móvil acetonitrilo-agua 75:25 1,5 mL/min. La acidez total titulable (ATT) se cuantificó a través de titulación ácido base con $\mathrm{NaOH}(0,1 \mathrm{~N})$, utilizando bureta digital y los sólidos solubles totales (SST, ${ }^{\circ}$ Brix) con un refractómetro digital marca Hanna (Hanna Instruments, Eibar, España) de rango 0 a $85 \%$ con precisión de $0,1^{\circ}$ Brix.
El análisis de los datos se realizó con el software Statistical Package for the Social Sciences (SPSS) v. 19, se determinaron las pruebas de normalidad (test de Shapiro-Wilk) y homogeneidad de varianzas (test de Levene), luego del cumplimiento de los anteriores supuestos se realizó un análisis de varianza y posteriormente la prueba de comparación de rangos múltiples de Tukey $(P \leq 0,05)$.

\section{RESULTADOS Y DISCUSIÓN}

Es importante mencionar que durante los $40 \mathrm{~d}$ almacenamiento refrigerado a $2^{\circ} \mathrm{C}$, los frutos de mandarina 'Arrayana' no presentaron síntomas de daños por frío visibles. Estos resultados concuerdan con los encontrados por Schirra y Cohen (1999), quienes mencionan que en algunos casos, los síntomas de daños por frío son visibles en los productos que han sido removidos del almacenamiento refrigerado a temperaturas superiores a las de almacenamiento. Por el contrario, Ghasemnezhad et al. (2008) reportaron daños por frío durante el almacenamiento refrigerado a $2^{\circ} \mathrm{C}$ en mandarina 'Satsuma'. Esto indica que hay factores genéticos que determinan la susceptibilidad a los daños por frío, pero sin olvidar también que dicha susceptibilidad también está dada por factores precosecha y manejo, durante el almacenamiento.

\section{Pérdida de peso}

Se presentó pérdida de peso en todos los tratamientos, con diferencias estadísticas $(P \leq 0,01)$ a partir de los $8 \mathrm{~d}$ de almacenamiento. Durante todo el almacenamiento la mayor pérdida de peso se obtuvo en los frutos sometidos a calentamiento intermitente de 8 d con una pérdida de $13,93 \pm 1,02 \%$ seguido muy de cerca del calentamiento intermitente cada $12 \mathrm{~d}$, mientras que los frutos sin tratamiento térmico (testigo) llegaron a una pérdida final del $8,43 \%$, sin diferencias estadísticas con los frutos sometidos a inmersión en agua caliente (Fig. 1). Resultados similares fueron encontrados por Hong et al. (2007), quienes mencionan que en mandarina 'Satsuma', el tratamiento con agua caliente no afectó la pérdida de peso.

La pérdida de agua por traspiración es la principal causa de disminución de peso de los productos vegetales en poscosecha, como segunda causa está la pérdida de materia seca por procesos respiratorios (Kader, 2002). Por lo que se puede inferir que en los frutos sometidos a calentamiento intermitente la transpiración 


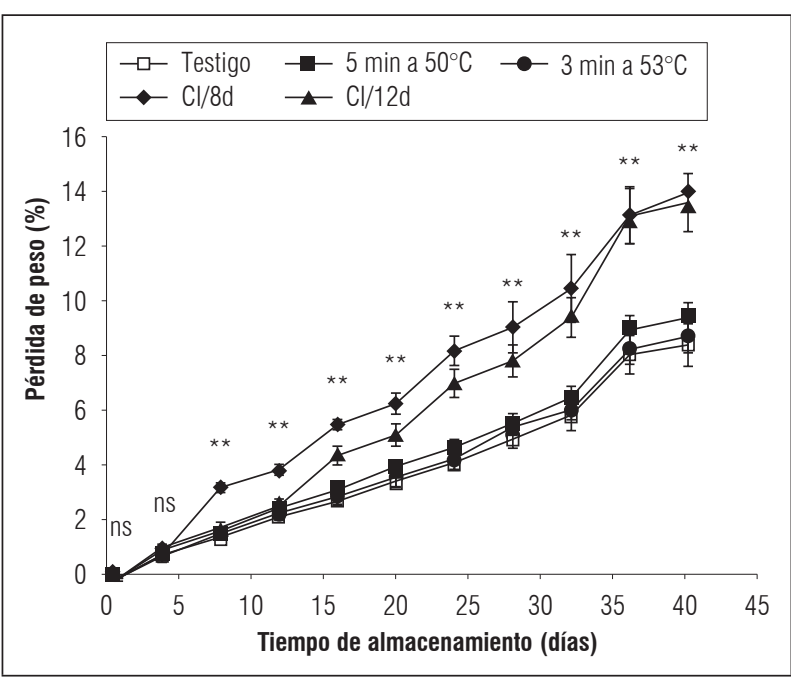

Figura 1. Pérdida de peso en frutos de mandarina 'Arrayana' almacenados a $2^{\circ} \mathrm{C}$ con diferentes tratamientos térmicos. Inmersión con agua caliente en tratamientos de 5 y $3 \mathrm{~min}$. Cl: calentamiento intermitente. ns: sin diferencia estadística; ** diferencias estadísticas al $1 \%$. Las barras indican error estándar.

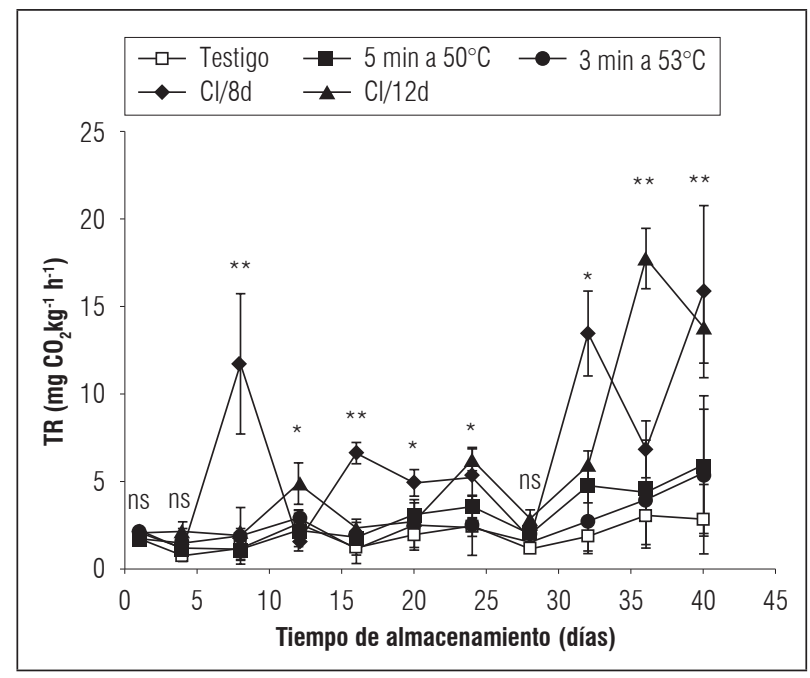

Figura 2. Tasa respiratoria (TR) de frutos de mandarina 'Arrayana' almacenados a $2^{\circ} \mathrm{C}$ con diferentes tratamientos térmicos. Inmersión con agua caliente en tratamientos de 5 y 3 min. Cl: calentamiento intermitente. ns: $s$ in diferencias estadísticas; * diferencias estadísticas al 5\%; ** diferencias estadísticas al 1\%. Las barras indican error estándar.

fue mayor, además de que presentó la mayor tasa respiratoria (Fig. 2) debido al traslado periódico de dichos frutos a temperatura ambiente, condición que aumenta la traspiración y respiración de los frutos. Pues según Hernández-Muñoz et al. (2008), la temperatura de almacenamiento determina la transpiración de los tejidos. Por esta razón, debe pensarse en mejorar la técnica del calentamiento intermitente, por ejemplo encerando los frutos, ya que de acuerdo con Kader (2002), la pérdida de agua es la principal causa del deterioro del fruto porque conlleva a la disminución directa del peso fresco y la calidad, asimismo, disminuye la vida en almacenamiento debido a que se aceleran los cambios durante la maduración y la senescencia.

Los resultados también se relacionan con lo observado por Bassal y El-Hamahmy (2011) quienes indican que los tratamientos térmicos pueden incrementar o disminuir las pérdidas de peso de los frutos, dependiendo del tratamiento y el producto agrícola, principalmente por la respuesta particular de cada fruto al tratamiento con calor.

\section{Tasa respiratoria}

Se presentaron diferencias estadísticas en casi todos los puntos de muestreo, con excepción de los días 1 , 4 y 28 de almacenamiento. Fue característico encontrar aumentos representativos en la tasa respiratoria de los frutos sometidos a calentamiento intermitente en los días que fueron expuestos a temperatura ambiente, lo cual también explica la mayor pérdida de peso en estos tratamientos. Los frutos de los demás tratamientos mantuvieron una tasa respiración más o menos estable durante el almacenamiento con un leve aumento después del día 28 (Fig. 2).

Estudios realizados por Balaguera-López et al. (2015) en frutos de uchuva, permitieron determinar que la respiración está relacionada con la temperatura, de tal manera que a mayor temperatura la respiración fue mayor, comportamiento que se ha encontrado en diferentes productos agrícolas y que explican lo ocurrido en los frutos de mandarina con los tratamientos de calentamiento intermitente, pues estos tratamientos a diferencia de los tratamientos con agua caliente e incluso el tratamiento testigo, periódicamente son sometidos a mayores temperaturas al retirarse de manera cíclica del almacenamiento refrigerado a temperatura ambiente. A pesar de que los frutos de mandarina son frutos no climatéricos, es importante mantener tasas respiratorias bajas para reducir la actividad metabólica del fruto, para conservar la calidad del fruto y retardar los procesos de senescencia (Pereira et al., 2013). En este orden de ideas, los 
tratamientos con agua caliente serían más ventajosos que el calentamiento intermitente por mantener tasas respiratorias más bajas, al parecer debido a que la alta temperatura del agua caliente puede reducir o eliminar la actividad enzimática, lo cual afecta la tasa respiratoria, tal como lo mencionan Alegria et al. (2012).

\section{Color y pigmentos}

El cambio de color de la epidermis en los frutos de mandarina mostró un aumento constante en función del tiempo de almacenamiento. Con la inmersión en agua caliente por $5 \mathrm{~min}$ a $50^{\circ} \mathrm{C}$ se encontró significativamente $(P \leq 0,05)$ menor al cambio de color, por otro lado, el calentamiento intermitente cada 8 d generó mayor delta de color en los frutos (Fig. 3). Este tratamiento también fue responsable de mayor contenido de carotenoides totales con un valor de 976,6 $\mu \mathrm{g} \mathrm{g} \mathrm{g}^{-1}$, también con diferencias significativas entre los tratamientos (Tab. 2). En relación a la clorofila total, los frutos de los tratamientos de inmersión en agua caliente durante 3 min a $53^{\circ} \mathrm{C}$ y de calentamiento intermitente cada $12 \mathrm{~d}$ presentaron la menor concentración de clorofilas, los frutos de los demás tratamientos fueron significativamente superiores (Tab. 2).

Los tratamientos térmicos con agua caliente tienen la posibilidad de afectar la síntesis de etileno, pues Lurie et al. (1996) afirman que con temperaturas superiores a $38^{\circ} \mathrm{C}$ se disminuyó la abundancia de mRNA de la enzima formadora de etileno ACC oxidasa. A esto se le suma el hecho de que el almacenamiento de los frutos de mandarina en dichos tratamientos fue continuo a $2^{\circ} \mathrm{C}$, por tanto, se esperaría que la producción de etileno fuera más baja que en los frutos con calentamiento intermitente, y como consecuencia los

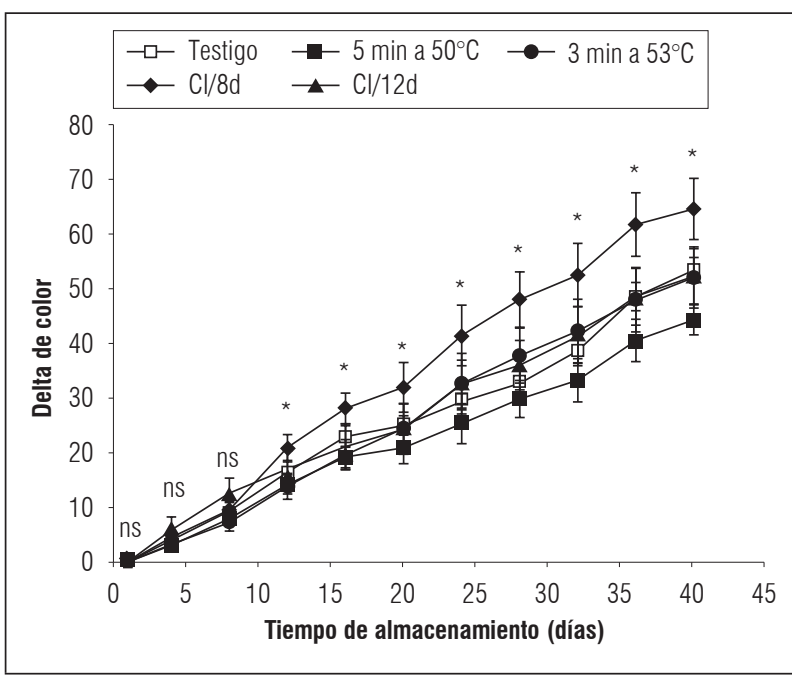

Figura 3. Delta de color en frutos de mandarina 'Arrayana' almacenados a $2^{\circ} \mathrm{C}$ con diferentes tratamientos térmicos. Inmersión con agua caliente en tratamientos de 5 y 3 min. Cl: calentamiento intermitente. ns: sin diferencias estadísticas; * diferencias estadísticas al $\mathbf{5 \%}$. Las barras indican error estándar.

cambios de color serán menores, tal como ocurrió con los frutos que fueron tratados con agua caliente durante $5 \mathrm{~min}$ a $50^{\circ} \mathrm{C}$. Al respecto, Rodrigo et al. (2013) mencionan que el etileno está involucrado en el cambio de color de la epidermis de cítricos.

Además, es posible que las fluctuaciones de temperatura presentadas en los tratamientos de calentamiento intermitente sean necesarios para la síntesis y actividad de enzimas involucradas en la biosíntesis de carotenoides y en la degradación de clorofilas, lo cual explicaría la mayor acumulación de carotenoides y la menor presencia de clorofilas en el tratamiento de calentamiento intermitente cada 8 d, así como el

Tabla 2. Parámetros de maduración de frutos de mandarina 'Arrayana' almacenados a $2^{\circ} \mathrm{C}$.

\begin{tabular}{|l|c|c|c|c|c|}
\hline $\begin{array}{c}\text { Tratamientos } \\
\text { térmicos }\end{array}$ & $\begin{array}{c}\text { Clorofila total } \\
\left(\mathrm{mg} \mathrm{g}^{-1}\right)\end{array}$ & $\begin{array}{c}\text { Carotenoides totales } \\
\left(\mu \mathrm{g} \mathrm{g}^{-1}\right)\end{array}$ & $\begin{array}{c}\text { Firmeza } \\
(\mathrm{N})\end{array}$ & $\begin{array}{c}\text { Acidez total titulable } \\
(\%)\end{array}$ & $\begin{array}{c}\text { Sólidos solubles totales } \\
\left({ }^{\circ} \text { Brix }\right)\end{array}$ \\
\hline Testigo & $0,10 \pm 0,01 \mathrm{a}$ & $715,14 \pm 70,39 \mathrm{~b}$ & $19,62 \pm 1,27 \mathrm{~b}$ & $0,67 \pm 0,13 \mathrm{~b}$ & $11,98 \pm 0,87 \mathrm{a}$ \\
\hline 5 min a $50^{\circ} \mathrm{C}$ & $0,09 \pm 0,008 \mathrm{a}$ & $572,45 \pm 73,12 \mathrm{~b}$ & $22,52 \pm 1,28 \mathrm{a}$ & $0,85 \pm 0,10 \mathrm{ab}$ & $11,35 \pm 0,46 \mathrm{a}$ \\
\hline 3 min a $53^{\circ} \mathrm{C}$ & $0,07 \pm 0,005 \mathrm{ab}$ & $760,93 \pm 64,91 \mathrm{~b}$ & $23,08 \pm 1,11 \mathrm{a}$ & $0,98 \pm 0,22 \mathrm{a}$ & $13,25 \pm 0,17 \mathrm{a}$ \\
\hline $\mathrm{Cl} / 8 \mathrm{~d}$ & $0,05 \pm 0,02 \mathrm{~b}$ & $976,60 \pm 52,88 \mathrm{a}$ & $18,98 \pm 0,67 \mathrm{~b}$ & $0,68 \pm 0,08 \mathrm{~b}$ & $11,25 \pm 0,68 \mathrm{a}$ \\
\hline $\mathrm{Cl} / 12 \mathrm{~d}$ & $0,06 \pm 0,006 \mathrm{~b}$ & $744,87 \pm 66,27 \mathrm{~b}$ & $19,16 \pm 0,95 \mathrm{~b}$ & $0,63 \pm 0,10 \mathrm{~b}$ & $11,28 \pm 1,08 \mathrm{a}$ \\
\hline Significancia & $*$ & $* *$ & $*$ & $n$ & $\mathrm{~ns}$ \\
\hline
\end{tabular}

ns: no hay diferencia estadística; * ${ }^{*}$ diferencias estadísticas al 1\%. Promedios con letras distintas en la misma columna, indican diferencia significativa según la prueba de Tukey $(P \leq 0,05)$. Inmersión con agua caliente en tratamientos de 5 y 3 min. Cl: calentamiento intermitente. 
mayor cambio de color de la epidermis (Tab. 2). Al respecto, Le Roux (2006) menciona la importancia de la fluctuación de la temperatura sobre el desarrollo del color en cítricos, y esta es una situación interesante para el proceso de comercialización, teniendo en cuenta que las mandarinas cultivadas en el trópico frecuentemente generan un pobre desarrollo del color, por lo que en muchos casos se opta por tratamientos de desverdizado con etileno (Rodrigo et al., 2013), por esta razón, es importante indicar que los tratamientos de calentamiento intermitente se convierten en una alternativa favorable como técnica poscosecha en las mandarinas arrayanas.

\section{Firmeza}

Con diferencias estadísticas $(P \leq 0,05)$, los frutos de mandarina de los tratamientos con agua caliente presentaron la mayor firmeza (Tab. 2). Estos resultados coinciden con lo encontrado en frutos de kiwi, donde la mayor firmeza se obtuvo con tratamientos térmicos de agua a 35 y $45^{\circ} \mathrm{C}$ (Ma et al., 2014). Los tratamientos de calentamiento intermitente no afectaron la firmeza en relación a los frutos testigo, situación que difiere de lo encontrado en frutos de Capsicum annuum donde el calentamiento intermitente redujo

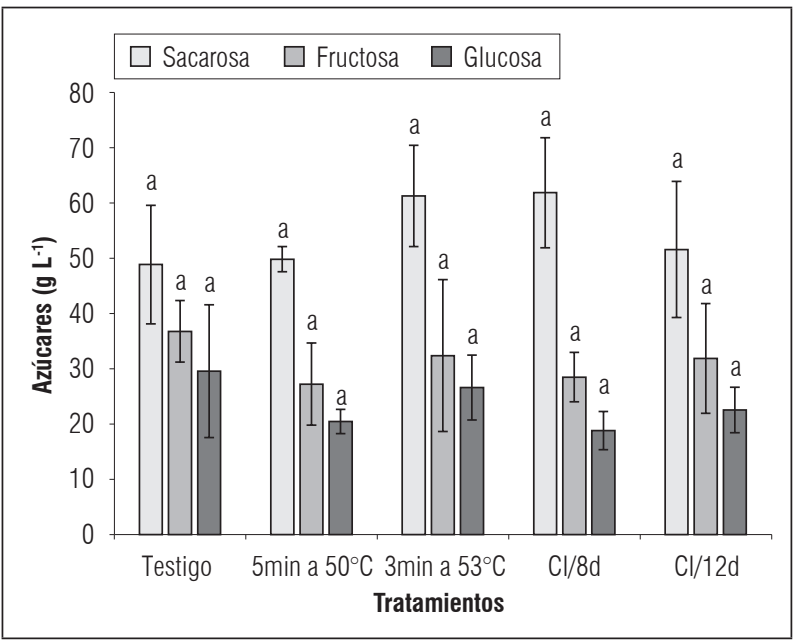

Figura 4. Contenido de azúcares solubles en frutos de mandarina 'Arrayana' almacenados a $2^{\circ} \mathrm{C}$ con diferentes tratamientos térmicos. Inmersión con agua caliente en tratamientos de 5 y $3 \mathrm{~min}$. Cl: calentamiento intermitente. Promedios con letras distintas en el tratamiento, indican diferencia significativa según la prueba de Tukey $(P \leq 0,05)$. Las barras indican error estándar. los daños por frío y mantuvo una mayor firmeza (Liu et al., 2015).

Varios estudios reportan que las altas temperaturas inhiben varias enzimas involucradas en el ablandamiento, por ejemplo, las enzimas $\beta$-mananasa y $\alpha$ y $\beta$-galactosidasa en tomates (Sozzi et al., 1997) y poligalacturonasa en mango (Ketsa et al., 1998). Esto puede ser debido a que las altas temperaturas pueden generar cambios en la conformación de proteínas (Paliyath et al., 2008), procesos que al parecer se presentaron en los frutos de mandarina sometidos a los tratamientos con agua caliente y explicarían la mayor firmeza en estos frutos.

\section{Azúcares y ácidos orgánicos}

No se observaron diferencias significativas entre tratamientos, los SST oscilaron en promedio entre $11,25 \pm 0,68$ y $13,25 \pm 0,17{ }^{\circ}$ Brix (Tab. 2), asimismo, para sacarosa, glucosa y fructosa tampoco hubo diferencias estadísticas (Fig. 4). Para la acidez total titulable, se presentaron diferencias estadísticas $(P \leq 0,05)$, se encontró que los frutos sometidos a inmersión en agua caliente durante 3 min a $53^{\circ} \mathrm{C}$ presentaron mayor ATT, mientras que los frutos testigos tuvieron menor ATT (Tab. 2), para el ácido oxálico también

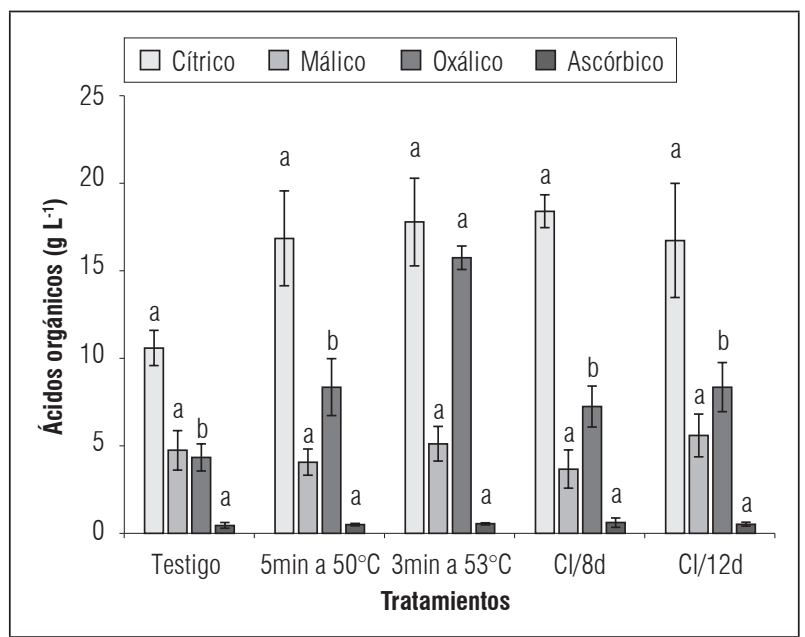

Figura 5. Contenido de ácidos orgánicos en frutos de mandarina 'Arrayana' almacenados a $2^{\circ} \mathrm{C}$ con diferentes tratamientos térmicos. Inmersión con agua caliente en tratamientos de 5 y 3 min. $\mathrm{Cl}$ : calentamiento intermitente. Promedios con letras distintas indican diferencia significativa entre el mismo ácido orgánico según la prueba de Tukey $(P \leq 0,05)$. Las barras indican error estándar. 
hubo diferencias estadísticas, donde el agua caliente a $53^{\circ} \mathrm{C}$ por 3 min generó mayor presencia de este ácido, los demás tratamientos tuvieron valores significativos más bajos. Los ácidos cítrico, málico y ascórbico no evidenciaron diferencias significativas (Fig. 5).

Varios autores reportan que los tratamientos con agua caliente incrementan los SST en frutos climatéricos (Liu et al., 2015; Lara et al., 2009), pero en los frutos de mandarina por su carácter no climatérico, es común que los parámetros relacionados con la madurez interna no cambien de manera representativa después de la cosecha, esto explicaría la no existencia de diferencias estadísticas en relación a los azúcares, a acidez y los ácidos orgánicos, excepto en el ácido oxálico. No obstante, Maldonado et al. (2004) indican que durante la refrigeración puede presentarse una disminución de los ácidos orgánicos, como indicativo de los cambios en el metabolismo energético, estabilidad del $\mathrm{pH}$ y compuestos de defensa que previenen o están implicados en la reparación de los daños causados por las bajas temperaturas, pero teniendo en cuenta que no hubo daños visibles por frío, incluso en los frutos testigo, se puede pensar que el metabolismo de los ácidos orgánicos pudo tener más implicación en los procesos propios de la maduración que en la respuesta a daños por frío en los diferentes tratamientos.

Por lo anterior, el mayor nivel de ácido oxálico en los frutos tratados con agua caliente a $53^{\circ} \mathrm{C}$ por $3 \mathrm{~min}$ puede estar asociado a cambios en la actividad enzimática del metabolismo de los ácidos orgánicos, o como intermediario metabólico en el proceso de maduración pero que no afecta la acidez total de los frutos. En concordancia, Ghasemnezhad et al. (2008) no observaron diferencias en la acidez de frutos de mandarina con tratamientos de agua caliente y Schirra et al. (2004) no encontraron efecto sobre los sólidos solubles totales, la acidez total titulable y el ácido ascórbico en frutos de naranja.

\section{CONCLUSIONES}

No se presentaron síntomas de daños por frío visibles en los frutos de mandarina arrayana almacenados a $2^{\circ} \mathrm{C}$ durante $40 \mathrm{~d}$. Al final del almacenamiento, los tratamientos térmicos utilizados no afectaron significativamente los azúcares, la ATT, los ácidos cítrico, málico y ascórbico ni los sólidos solubles totales, teniendo poco efecto sobre la madurez interna de los frutos de mandarina. El calentamiento intermitente se caracterizó por aumentar las pérdidas de peso de los frutos, la tasa respiratoria pero generó mayor cambio de color de la epidermis al presentar mayor acumulación de carotenoides totales y una baja concentración de clorofilas, siendo este último, un resultado importante desde el punto de vista comercial porque las mandarinas arrayanas comúnmente presentan pobre desarrollo del color, además, es un resultado que deja abierta la posibilidad de seguir trabajando con el calentamiento intermitente pero con algunas modificaciones para lograr optimizarlo.

\section{AGRADECIMIENTOS}

Los autores agradecen a la "Vicerrectoría de Investigaciones, Universidad el Bosque, Bogotá (Colombia), por la financiación de esta investigación a través del proyecto PCI-2015-8256.

Conflicto de intereses: el manuscrito fue preparado y revisado con la participación de los autores, quienes declaran no tener algún conflicto de interés que coloquen en riesgo la validez de los resultados aquí presentados.

\section{REFERENCIAS BIBLIOGRÁFICAS}

Alegria, C., J. Pinheiro, M. Duthoit, E.M. Gonçalves, M. Moldão-Martins y M. Abreu. 2012. Fresh-cut carrot (cv. Nantes) quality as affected by abiotic stress (heat shock y UV-C irradiation) pretreatments. Food Sci. Technol-Leb. 48(2), 197-203. Doi: 10.1016/j. lwt.2012.03.013

Artés, F. y F. Artés-Hernández. 2002. Daños por frío en la postrecolección de frutas y hortalizas. pp. 299-310. En: López, A., A. Esnoz y F. Artés (eds.). Avances en Ciencias y Técnicas del Frío-1. Universidad Politécnica; Sociedad Española de Ciencias y Técnicas del Frío, Cartagena, España.

Artés, F., P. Velasquez y J.G. Marin. 1998. Reduction of decay and chilling injuries in cold stored oranges. pp. 243-248. En: Bertolini, P., P.C. Sijsmons, M.E. Guerzoni y F. Serra. (eds.). Nom conventional methods for the control de postharvest disease and microbial spoilage. Ed. European Comission, Bruselas, Bélgica.

Balaguera, H.E. 2015. Comportamiento poscosecha del fruto de uchuva (Physalis peruviana L.): efecto del 1-metilciclopropeno y de refrigeración. Tesis de doctorado. Facultad de Ciencias Agrarias, Universidad Nacional de Colombia, Bogotá, Colombia.

Balaguera-López, H.E., C.A. Martínez y A. Herrera. 2015. Refrigeration affects the postharvest behavior of 1-methylcyclopropenetreated cape gooseberry (Physalis 
peruviana L.) fruits with the calyx. Agron. Colomb. 33(3), 356-364. Doi: 10.15446/agron.colomb. v33n3.51896

Balaguera-López, H., M. Espinal-Ruiz, L. Zacarías y A. Herrera. 2016. Effect of ethylene and 1-methylcyclopropene on the postharvest behavior of cape gooseberry fruits (Physalis peruviana L.). Food Sci. Technol. Int. 23(1), 86-96 Doi: 10.1177/1082013216658581

Bassal, M. y M. El-Hamahmy. 2011. Hot water dip and preconditioning treatments to reduce chilling injury and maintain postharvest quality of Navel and Valencia oranges during cold quarantine. Postharvest Biol. Technol. 60, 186-191. Doi: 10.1016/j. postharvbio.2011.01.010

Carvalho, C., P. Navarro y A. Salvador. 2012. Poscosecha. pp. 223-284. En: Garcés, L. y C. Pássaro (eds.). Cítricos: cultivo, poscosecha e industrialización. Editorial Artes y Letras, Itagüí, Colombia.

Chaparro-Zambrano, H., H. Velásquez-Ramírez y J.O. Ordúz-Rodríguez. 2017. Evaluation of 'Arrayana' tangerine (Citrus reticulata Blanco) grafted onto different rootstocks in tropical lowlands of Colombian Orinoquia, 2005-2011 (second cycle). Agron. Colomb. 35(1), 29-34. Doi: 10.15446/agron.colomb.v35n1.60082

Crisosto, C.H., B. Mitcham y M. Cantwell. 2008. Optimum temperature conditions for produce handlers. Central Valley Postharvest Newsletter No. 17 Cooperative Extension, University of California, Davis, CA, USA.

Fallik, E. 2004. Prestorage hot water treatments (immersion, rinsing and brushing). Postharvest Biol. Technol. 32, 125-134. Doi: 10.1016/j.postharvbio.2003.10.005

Ghasemnezhad, M., K. Marsh, R. Shilton, M. Babalar y A. Wolf. 2008. Effect of hot water treatments on chilling injury and heat damage in 'satsuma' mandarins: Antioxidant enzymes and vacuolar ATPase, and pyrophosphatase. Postharvest Biol. Technol. 48, 364-371. Doi: 10.1016/j.postharvbio.2007.09.014

Hernández-Muñoz, P., E. Almenar, V. Del Valle, D. Vélez y R. Gavara. 2008. Effect of chitosan coating combined with postharvest calcium treatment on strawberry (Fragaria $x$ ananassa) quality during refrigerated storage. Food Chem. 110, 428-435. Doi: 10.1016/j. foodchem.2008.02.020

Holland, N., F.L. Nunes, I.U. de Medeiros y M.T. Lafuente. 2012. High-temperature conditioning induces chilling tolerance in mandarin fruit: a cell wall approach. J. Sci. Food Agric. 92, 3039-3045. Doi: 10.1002/jsfa.5721

Hong, S., H. Lee y D. Kim. 2007. Effects of hot water treatment on the storage stability of Satsuma mandarin as a postharvest decay control. Postharvest Biol. Technol. 43, 271-279. Doi: 10.1016/j.postharvbio.2006.09.008

Kader, A.A. 2002. Postharvest technology of horticultural crops. $3^{\text {rd }}$ ed. Division of Agriculture and Natural Resources, University of California, Oakland, CA, USA.
Ketsa, S., S. Chidragool, J.D. Klein y S. Lurie. 1998. Effect of heat treatment on changes in softening, pectic substances and activities of polygalacturonase, pectinesterase and $\beta$-galactosidase of ripening mango. J. Plant Physiol. 153, 457-461. Doi: 10.1016/ S0176-1617(98)80174-0

Ladaniya, M. 2008. Citrus fruit: biology technology and evaluation. Elsevier, Atlanta, GA. Doi: 10.1016/B9780-12-374130-1.X5001-3

Lado, J., O. Cronje, M.J. Rodrigo y L. Zacarías. 2015. Resistance to chilling injury in red, lycopene-accumulating tissue of cold-stored grapefruits. Acta Hortic. 1079, 249-256. Doi: 10.17660/ActaHortic.2015.1079.29

Lafuente, M.T. y L. Zacarías. 2006. Postharvest physiological disorder in citrus fruit. Stewart Postharvest Rev. 2, 1-9. Doi: 10.2212/spr.2006.1.2

Lara, M.V., J. Borsani, C.O. Budde, M.A. Lauxmann, V.A. Lombardo, R. Murray, C.S. Andreo y M.F. Drincovich. 2009. Biochemical and proteomic analysis of 'Dixiland' peach fruit (Prunus persica) upon heat treatment. J. Exp. Bot. 60, 4315-4333. Doi: 10.1093/jxb/erp267

Le Roux, S. 2006. Preharvest manipulation of rind pigments of Citrus spp. Tesis de maestría. Department Horticultural Sciences, University of Stellenbosch, Stellenbosch, Sudáfrica.

Liu, L., Y. Wei, F. Shi, C. Liu, X. Liu y S. Ji. 2015. Intermittent warming improves postharvest quality of bell peppers and reduces chilling injury. Postharvest Biol. Technol. 101, 18-25. Doi: 10.1016/j.postharvbio.2014.11.006

Lurie, S., A. Handros, E. Fallik y R. Shapira. 1996. Reversible inhibition of tomato fruit gene expression at high temperature. Plant Physiol. 110, 1207-1214.

Ma, Q., S. Jiangtao, D. Huber, X. Dong, Y. Han, Z. Zhang y J. Rao. 2014. Effect of hot water treatments on chilling injury and expression of anew C-repeat binding factor (CBF) in 'Hongyang' kiwifruit during low temperature storage. Postharvest Biol. Technol. 97, 102110. Doi: 10.1016/j.postharvbio.2014.05.018

Maldonado, R.M., M.T. Sánchez-Ballesta, R. Alique, I. Escribano y C. Merodio. 2004. Malate metabolism and adaptation to chilling temperature storage by pretreatment with high $\mathrm{CO}_{2}$ levels in Annona cherimola fruit. J. Agric. Food Chem. 52, 4758-4763. Doi: 10.1021/jf0353842

Mendoza, F., P. Dejmek y J.M. Aguilera. 2006. Calibrated color measurements of agricultural foods using image analysis. Postharvest Biol. Technol. 41, 285-295. Doi: 10.1016/j.postharvbio.2006.04.004

Ordúz-Rodríguez, J.O., J. Monroy, S. Barrera, V. Núñez y G.A. Ligarreto. 2012. Caracterización morfo-agronómica y molecular de mandarina 'Arrayana' en el piedemonte del Meta (Colombia). Corpoica Cienc. Tecnol. Agropecu. 13, 5-12. Doi: 10.21930/rcta. vol13_num1_art:234 
Paliyath, G., D.P. Murr, A.K. Handa y S. Lurie. 2008. Postharvest biology and technology of fruits, vegetables, and flowers. Wiley-Blackwell Publishing, Nueva Delhi, India.

Pereira, D., M. Rodrigues, J. Da Costa, R. Pires, Y. Bruckner y C. Horst. 2013. Cold storage of peaches cv. Aurora grown in the Zona da Mata Mineira, Minas Gerais State, Brazil. Rev. Ceres 60(6), 833-841. Doi: 10.1590/ S0034-737X2013000600012

Rodrigo, M., B. Alquézar, E. Alós, J. Lado y L. Zacarías. 2013. Biochemical bases and molecular regulation of pigmentation in the peel of Citrus fruit. Sci. Hortic. 163, 46-62. Doi: 10.1016/j.scienta.2013.08.014

Rozenzvieg, D., C. Elmaci, A. Samach, S. Lurie y R. Porat. 2004. Isolation of four heat shock protein cDNAs from grapefruit peel tissue and characterization if their expression in response to heat and chilling temperature stresses. Physiol. Plant. 121, 421-428. Doi: 10.1111/j.1399-3054.2004.00334.x

Sala, J.M. y M.T. Lafuente. 2000. Catalase enzyme activity is related to tolerance of mandarin fruits to chilling.
Postharvest Biol. Technol. 20, 81-89. Doi: 10.1016/ S0925-5214(00)00115-0

Schirra, M. y E. Cohen. 1999. Long-term storage of 'Olin$\mathrm{da}$ ' oranges under chilling and intermittent warming temperatures. Postharvest Biol. Technol. 16, 63-69. Doi: 10.1016/S0925-5214(98)00097-0

Schirra, M., M. Mulas, A. Fadda y E. Cauli, 2004. Cold quarantine responses of blood oranges to postharvest hot water and hot air treatments. Postharvest Biol. Technol. 31, 191-200. Doi: 10.1016/j.postharvbio.2003.09.002

Shadmani, N., S.H. Ahmad, N. Saari, P. Ding y N.E. Tajidin. 2015. Chilling injury incidence and antioxidant enzyme activities of Carica papaya L. 'Frangi' as influenced by postharvest hot water treatment and storage temperature. Postharvest Biol. Technol. 99, 114-119. Doi: 10.1016/j.postharvbio.2014.08.004

Sozzi, G.O., O. Cascone y A.A. Fraschina. 1997. Effect of high temperature stress on endo- $\beta$-mannanase and $\alpha$ - and $\beta$-galactosidase activities during tomato fruit ripening. Postharvest Biol. Technol. 9, 49-63. Doi: 10.1016/0925-5214(96)00014-2 